\title{
ESTUDIO DE ISÓPODOS TERRESTRES (CRUSTACEA: ISOPODA: ONISCIDEA) EN TRES LOCALIDADES DE BOYACÁ, COLOMBIA
}

\section{STUDY OF TERRESTRIAL ISOPODS (CRUSTACEA: ISOPODA: ONISCIDEA) IN THREE LOCALITIES OF BOYACA, COLOMBIA}

\author{
Ariel Fernando Preciado; John Wilson Martinez ${ }^{2}$
}

Fecha de recepción: Abril 11 de 2014 Fecha de aceptación: Septiembre 14 de 2014

\begin{abstract}
RESUMEN
Se analizó la composición y abundancia de isopodos terrestres, mediante colecta directa, en tres localidades del departamento de Boyacá, Colombia; Tinjacá, Tibasosa y Tota. Se identificaron cinco especies distribuidas en dos familias para un total de 2516 individuos colectados, de los cuales se registraron por la primera vez en Boyacá las espeicies; Porcellio dilatatus (Brandt), Porcellionides pruinosus (Brandt), Armadillidium vulgare (Latreille) y Armadillidium nasatum (Budde-Lund). Se hallaron diferencias estadísticamente significativas respecto a: número de individuos, localidad y especies presentes. La mayor abundancia y riqueza se encontró en el municipio de Tibasosa. El mircrohábitat con mayor predominancia de los isópodos muestreadosfue el denominado plateo o suelo circundante a las plantas. La especie más abundante fue P. scaber y la especie más común fue A. vulgare. Las variables ambientales evaluadas no se relacionaron con el número de individuos colectados.
\end{abstract}

Palabras clave: Crustacea, oniscidea, Boyacá, composición, abundancia.

1 Biólogo, Universidad Pedagógica y Tecnológica de Colombia, Tunja. Colombia. apreciado.fr@gmail.com

2 Docente-investigador, I. A. MS.c en Ciencias agrarias énfasis Entomología. Universidad Pedagógica y Tecnológica de Colombia Tunja.Colombia.john.martinez@uptc.edu.co 


\begin{abstract}
The composition and abundance of terrestrial isopods were analyzed by direct collection at three localities in the department of Boyacá, Colombia: Tinjacá, Tibasosa, and Tota. Five species distributed in two families were identified from a total of 2516 field collected individuals, for which new occurrence reports in Boyacá are presented for: Porcellio dilatatus (Brandt), Porcellionides pruinosus (Brandt), Armadillidium vulgare (Latreille) and Armadillidium nasatum (Budde-Lund). Statistically significant differences were found regarding the number of individuals, locality, and species present. The greatest abundance and richness were found in the municipality of Tibasosa. The isopods sampled were mainly found in the so-called 'plateo' microhabitat, or the surrounding soil of the vegetation. The most abundant species was $P$. scaber and the most common one was A. vulgare. The environmental conditions were not related tothe number of individuals collected.
\end{abstract}

Keywords: Crustacean, Oniscidea, Boyacá, composition, abundance.

\section{INTRODUCCIÓN}

Los isópodos terrestres habitan desde la zona supralitoral, bosques, cordilleras, montañas, agroecosistemas, cavernas subterráneas, hasta desiertos (Paoletti y Hassall, 1999). Se considera que varias especies participan en el ciclaje de nutrientes (Hunter et al., 2003; Leistikow, 2001; Leistikow y Araujo, 2006; Quadros y Araujo, 2007) y al ser detritívoros (Leistikow y Araujo, 2006) contribuyen con esto en la fauna del suelo (Lopes et al., 2005). Sin embargo, estos organismos se han observado consumiendo raíces tiernas en Rosáceas y Mirtáceas, al igual que en hojas de hortalizas, causando debilitamiento, hojas con baja turgencia, decaimiento, vuelco, quiebres, daños en la emergencia de plántulas y daños en cotiledones y tallos (Saluso, 2001). Algunos producen heridas que se concentran en el segmento inferior del tallo en forma transversal y longitudinal (Saluso, 2001; González, 2004). La especie Oniscus asellus se ha mencionado como plaga en el cultivo de cebolla de bulbo en los municipios de Cucaita y Tibasosa en el departamento de Boyacá (Viteri et al., 2005).
La fauna de isópodos terrestres de Sur América ha sido poco estudiada (Leistikow y Araujo, 2006); en Colombia el estudio de Oniscidea ha sido escaso, y debido a que están tomando importancia económica y fitosanitaria al causar daños serios en plantas cultivadas, surge la necesidad de iniciar estudios en el departamento, identificando plantas hospederas y reconociendo las especies de isópodos presentes en las localidades de: Funza Abajo (Tinjacá), Patrocinio (Tibasosa) y Guaquira (Tota). Todo ello sirve para contribuir en la integración de metodologías para estructurar un programa MIP (Manejo Integrado de Plagas), que a mediano y largo plazo brinde a los agricultores un conjunto de técnicas con principios de sostenibilidad, competitividad y equidad.

\section{MATERIALES Y METODOS}

\section{Sitios de estudio}

Este estudio se llevó a cabo en el departamento de Boyacá, sobre la cordillera Oriental Colombiana (Fig.1), comprendido en tres localidades 
(Tab.1): la zona 1, situada entre las coordenadas 053' 45.3" N y 7339'40.6" NO, en el municipio de Tinjacá, vereda Funza Abajo, finca Las Delicias, con plantaciones de Prunus persica, $2150 \mathrm{~m}$ de altura; La zona 2 ubicada entre las coordenadas $05^{\circ} 45^{\prime} 17.4^{\prime \prime} \mathrm{N}$ y $72^{\circ} 58^{\prime} 38.0^{\prime \prime} \mathrm{NO}$, el municipio de Tibasosa, vereda Patrocinio, finca Puerto Arturo, con cultivos de lechuga Lactuca sativa L. y cebolla cabezona Allium cepa L., $2470 \mathrm{~m}$ de altura; y la zona 3 de predominio forestal Alnus sp. y Eucalyptus sp., situada entre $05^{\circ} 33^{\prime} 53.2^{\prime \prime} \mathrm{N} \mathrm{y}$ $72^{\circ} 59^{\prime} 10.8^{\prime \prime} \mathrm{NO}$, municipio de Tota, vereda Guáquira, $2850 \mathrm{~m}$ de altura.

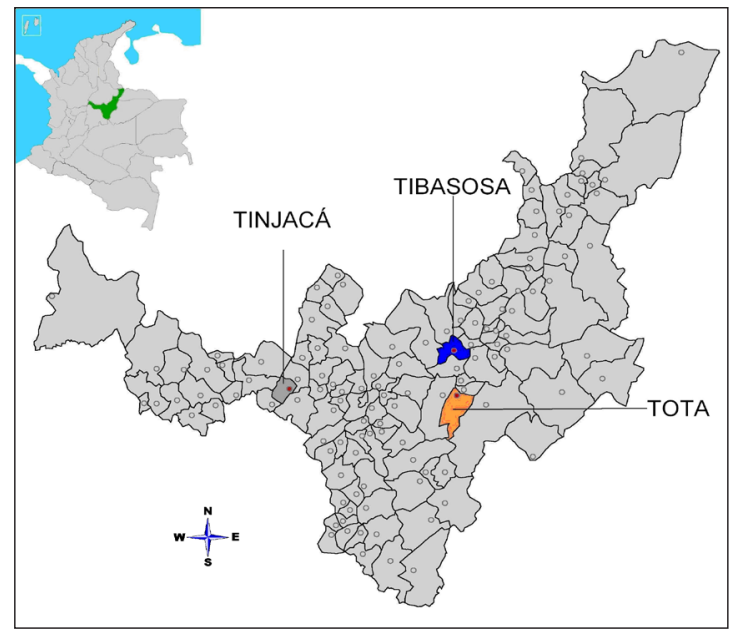

Figura 1. Ubicación geográfica de las zonas de muestreo en el departamento de Boyacá, Colombia

\section{Método de muestreo}

Se realizó una única colecta en cada localidad, con un esfuerzo de colecta de 4 horas/hombre/día, seleccionándose aleatoriamente tres localidades del departamento de Boyacá. Cada zona con un área aproximada de 4 hectáreas, en cada una de las cuales se estableció un área efectiva de $1,000 \mathrm{~m}^{2}$ y se tomaron 5 muestras aleatorias, empleando un cuadrante de $50 \mathrm{~cm}$ $x 50 \mathrm{~cm}$.

Los isópodos encontrados en cada cuadrante se colectaron mediante captura directa con pinzas entomológicas y luego se ubicaron en recipientes plásticos, colocando previamente material vegetal que les sirviera de alimento y les garantizara las condiciones apropiadas para su supervivencia durante el traslado al laboratorio de la UPTC.

En el momento de la colecta, se tomó información de temperatura ambiente (Datalogger USB - Extech Instruments), temperatura del suelo (geotermómetro Hanna Instruments), humedad relativa (Datalogger USB - Extech Instruments) y altitud (Garmin E-trex Handheld GPS); así mismo se definieron los micro hábitats en donde se encontraron las cochinillas de humedad.

\section{Procedimiento en laboratorio}

El análisis y observación del material recolectado se llevó a cabo en el Laboratorio de Control Biológico del Grupo Manejo Biológico de Cultivos de la Universidad Pedagógica y Tecnológica de Colombia. Se hizo un recuento de especimenes colectados por replica y por lugar, cuyos ejemplares se dejaron almacenados y conservados en el laboratorio antes mencionado.

Tabla 1. Características de los tres municipios del departamento de Boyacá, en las cuales se realizó el muestreo de cochinillas de humedad

\begin{tabular}{l|c|c|c|l}
\hline Municipio & $\begin{array}{c}\text { Altitud } \\
(\mathbf{m s n m})\end{array}$ & $\begin{array}{c}\text { Temperaturas } \\
\left({ }^{\circ} \text { centígrados) }\right.\end{array}$ & $\begin{array}{c}\text { Humedad } \\
\text { Relativa }\end{array}$ & Zona de vida \\
\hline Tinjacá & 2175 & 22 & 60 & Bosque Húmedo Montano Bajo \\
\hline Tibasosa & 2506 & 21 & 65 & Bosque Húmedo Montano Bajo \\
\hline Tota & 2870 & 16 & 70 & Bosque Húmedo Montano \\
\hline
\end{tabular}


Preservación: Los especímenes se preservaron en alcohol al 70\% con algunas gotas de glicerina; otros se clarificaron con $\mathrm{KOH}$ y luego se preservaron en alcohol al $70 \%$.

Identificación taxonómica: Se utilizó estereoscopio de luz (Motic SMZ-168 con 7.5X-50X) y claves taxonómicas para isópodos terrestres de Vandel (1960), Hopkin (1991) y Araujo et al., (1996).

\section{Análisis de datos}

Se empleó la prueba Chi-cuadrado para determinar si había diferencias, entre la cantidad de individuos que hay por especie en cada uno de los sitios, entre el número de individuos de cada especie, entre la cantidad de individuos respecto a la altitud del sitio muestreado (Tab. 2).

Se utilizó el Coeficiente de correlación de Spearman (Software R 3.0) de cada una de las variables analizadas. Se correlacionaron la temperatura del suelo, la temperatura ambiente y la humedad relativa con el número de individuos hallado por cada lugar, para determinar si existía alguna relación entre estas variables.

A partir de allí, se establecieron conclusiones con cada variable en cada lugar para determinar su efecto en el número de individuos de isópodos presentes.

\section{RESULTADOS Y DISCUSIÓN}

\section{Determinación de especies}

El muestreo permitió obtener un total de 2.516 individuos colectados en las tres localidades y distribuidos en cinco especies: Porcellio scaber (Latreille), Porcellio dilatatus (Brandt), Porcellionides pruinosus (Brandt), Armadillidium vulgare (Latreille) y Armadillidium nasatum (Budde-
Lund) siendo los primeros registros de ocurrencia para Boyacá.

P. scaber es un habitante abundante en el estrato de descomposición de bosques, nativo de Euro$\mathrm{pa}$, excepto en el sureste y fue introducido por el hombre en gran parte del mundo, principalmente en áreas urbanas. Se encuentra igualmente al norte de los EUA y en el estado de Texas, en Canadá, México, Antillas, Argentina (Buenos Aires), Brasil (Rio Grande do Sul), Chile (Isla Juan Fernández). En México se registra para los estados de Veracruz, Puebla y Michoacán (Rodríguez et al., 2014; Leistikow y Wolfgang, 1999).

Armadillidium vulgare se considera nativa de la región Mediterránea de Europa e introducida en gran parte del mundo como Canadá (Ontario), EUA (Columbia Británica, Arkansas, Michigan, Georgia, Texas), Brasil (Sao Paulo), Chile (Isla Juan Fernandez), Uruguay (Montevideo), Argentina (Buenos Aires) y Bermuda. En México se registra para México, D. F. y Morelia, Michoacán (Rodríguez et al., 2014; Leistikow y Wolfgang, 1.999).

Porcellionides pruinosus es de origen mediterráneo, sinantrópica cosmopolita, ampliamente distribuida en América del Sur, América Central, México y las Antillas, Bermudas, islas Galápagos y Ecuador; igualmente en la mayor parte de EUA y Canadá. En México, P. pruinosus fue registrada en el Distrito Federal, Atepec cerca de Ixtlán y Yucatán (Rodríguez et al., 2014).

Porcellio dilatatus es una especie del género Porcellio, perteneciente a la familia Porcellionidae que tiene una amplia distribución en Europa y ha sido introducida a regiones como Norte America en Canada y EUA (Washington, Arizona y California), Australia y Brasil (Minas Gerais hasta Rio Grande do Sul) desde su sitio de origen en Europa Occidental (Houston y Beesley, 2002; Leistikow y Wolfgang, 1999). 
Armadillidium nassatum se presenta esporádicamente a lo largo del sur de Inglaterra y Gales. Es característica de hábitats con vegetación poco densa, tanto natural como sinantrópica sometida a altos niveles de insolación (Gregory, 2009). Igualmente se ha reportado esta especie originaria del suroeste de Europa, en Norteamerica en alguna zonas del Noreste y Arkansas (Myers et al., 2015) y en Brasil (Rio Grande Do Sul) (Leistikow y Wolfgang, 1999).

La distribución de isópodos terrestres en el nuevo mundo ha sido documentada muy poco, siendo la más reciente la realizada por Leistikow y Wolfgang (1.999); para Colombia en particular no existen reportes sistematizados sobre presencia de isópodos por zonas o rangos altitudinales.

\section{Composición de la isopodofauna}

Abundancia: Se encontraron diferencias estadísticamente significativas respecto al número de individuos que hay por especie en cada zona muestreada (Tab.2), en Tinjacá y Tota se encuentra mayor número de individuos de $A$. vulgare y en Tibasosa se encuentra principalmente la especie $P$. scaber.

La mayor abundancia se encuentra en Tibasosa con 1827 individuos distribuidos en 5 especies (Porcellio scaber, Porcellio dilatatus, Armadillidium vulgare, Armadillidium nasatum y Porcellionides pruinosus), seguido por la localidad Tinjacá con 575 individuos de dos especies ( $P$. dilatatus y $A$. vulgare) y Tota con 114 individuos distribuidos en dos especies (Tab.2, Fig.2A).

De esta forma la especie abundante fue P. scaber, con mayor número de individuos capturados y mayor número de individuos por localidad (1611); la especie común en las tres zonas fue A. vulgare (Tab.2).

Riqueza de especies: El mayor número de especies se encuentra en Tibasosa presentando 5 especies, seguido por Tinjacá, con las especies $P$. dilatatus y A. vulgare; y Tota, con dos especies, P. scaber y A. vulgare (Fig.2B).

Tabla 2. Número total de individuos de las especies de isópodos terrestres presentes en las localidades de estudio

\begin{tabular}{l|c|c|c}
\hline & Tinjacá (2150m) & Tibasosa (2470m) & Tota (2850m) \\
\hline Porcellio scaber & - & 1611 & 36 \\
\hline Porcellio dilatatus & 41 & 22 & - \\
\hline Porcellionides pruinosus & - & 22 & - \\
\hline Armadillidium vulgare & 534 & 47 & 78 \\
\hline Armadillidium nasatum & - & 125 & - \\
\hline Total & 575 & 1827 & 114 \\
\hline Chi cuadrado & 422,693 & 5327,140 & p: $8,366 \mathrm{e}-05$ \\
Número individuos & p: $6,325 \mathrm{e}-94$ & $\mathrm{p}: 0$ & \\
\hline Chi cuadrado 0 & & 1873,7580 & \\
altitud & & $\mathrm{p}: 0$ & \\
\hline \multirow{2}{*}{ Chi cuadrado especie } & & p: 0 & \\
& & & \\
\hline
\end{tabular}




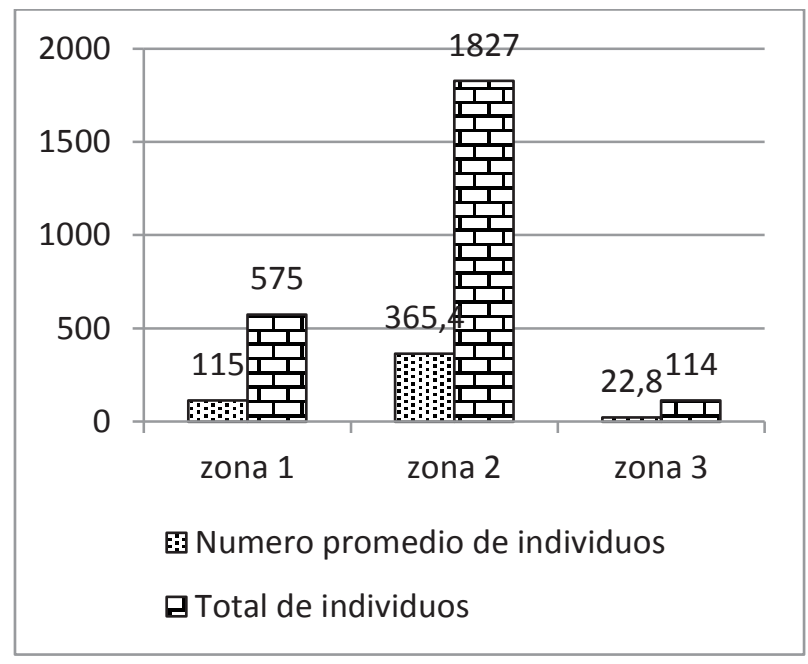

A

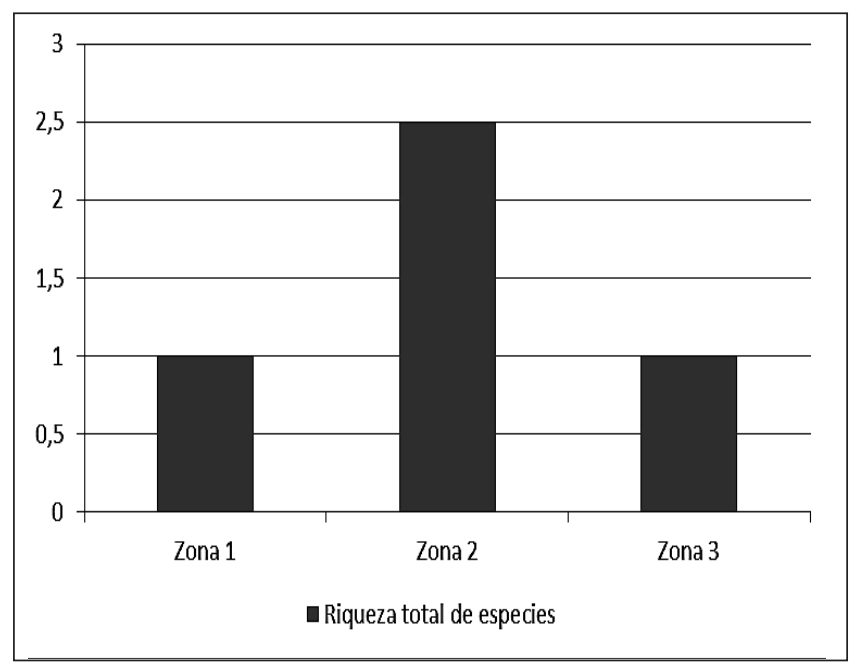

B

Figura 2. Abundancia promedia y total de isópodos por cada zona muestreada (A) y Riqueza total de especies de isópodos presentes (B). Zona 1. Tinjacá, Zona 2. Tibasosa, Zona Tota

Microhábitats: En las zonas de muestreo se identificaron cinco tipos de microhábitats los cuales se definieron como: 1) Plateo, 2) Zanja de Riego, 3) Tronco caído (en descomposición), 4) Bajo piedras, 5) Bajo terrones húmedos.

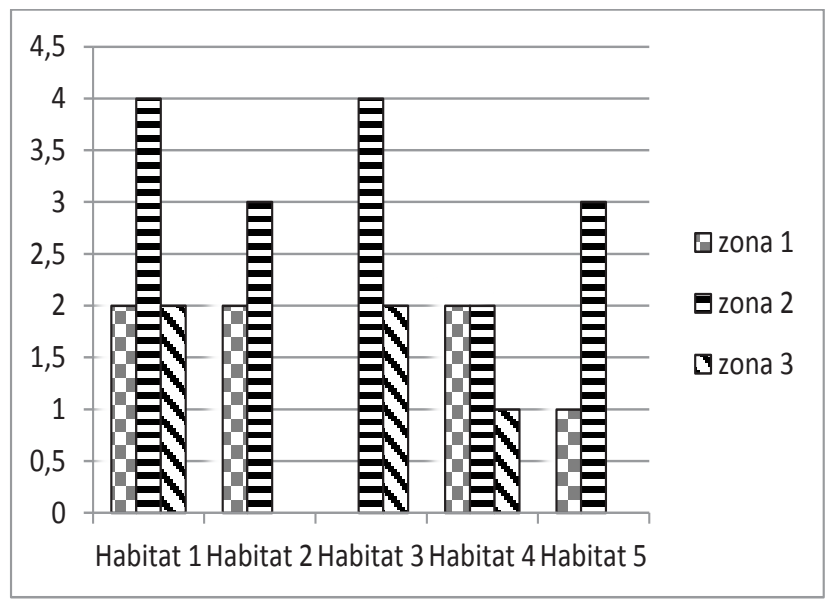

A
En Tibasosa se halló el mayor número de microhábitats y en menor grado las zonas de Tinjacá y Tota, respectivamente (Fig.3A). Igualmente el mayor número de microhábitats presentes en las zonas de estudio fue Plateo, seguido del microhábitat tronco caído (Fig.3B).

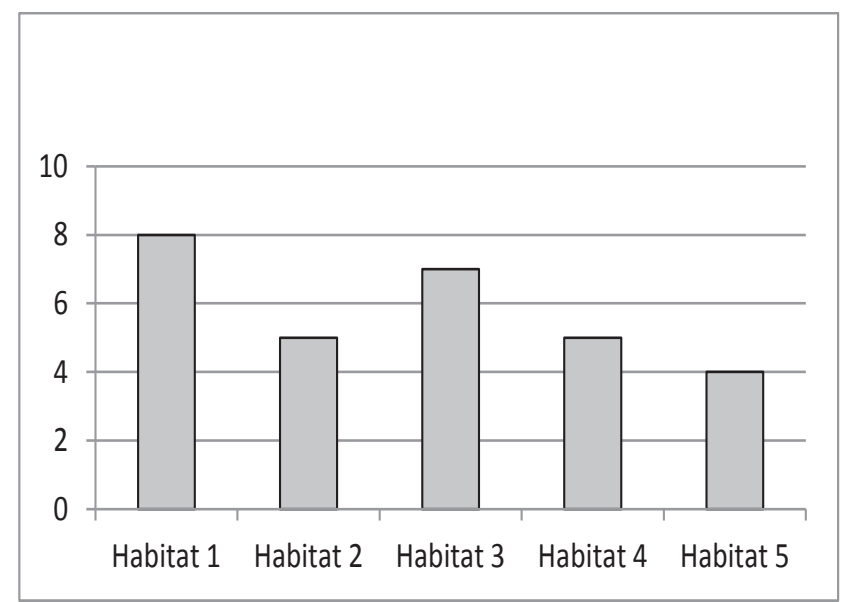

B

Figura 3. Número de especies de isópodos presentes por microhábitats en cada zona. Zona 1. Tinjacá, Zona 2. Tibasosa, Zona 3 Tota. (A) y número total de microhábitats encontrados 1. Plateo, 2. Zanja de Riego, 3. Tronco caído (en descomposición), 4. Bajo piedras, 5. Bajo terrones húmedos. (B). 
Influencia de variables evaluadas: Las variables de temperatura del suelo, temperatura ambiente y humedad relativa (Tab.3), no se relacionan con el número de individuos encontrados, al igual que no hay relación entre cuadrantes por cada zona (Tab.4). Es probable que las condiciones o características propias del suelo (como humedad del suelo, nivel freático, tipo de suelo, etc) sean más determinantes, sobre la cantidad de individuos de "Cochinillas de humedad" que se pueden encontrar en un hábitat determinado, que las condiciones ambientales aquí evaluadas. Un próximo trabajo, debería involucrar aspectos propios del suelo como los antes mencionados, que podrían tener una relación más estrecha e indicar factores que promuevan o restrinjan el tamaño de las poblaciones de "Cochinillas de humedad" en un suelo dado.

Tabla 3. Variables ambientales registradas en cada cuadrante de suelo muestreado. $\mathrm{T}^{\circ} \mathrm{s}$ : temperatura del suelo; $\mathrm{T}^{\circ}$ a: temperatura ambiente; Hr: Humedad relativa.

Zona 1. Tinjacá, Zona 2. Tibasosa, Zona 3 Tota.

\begin{tabular}{c|c|c|c|c|c|c|c|c|c}
\hline \multirow{2}{*}{$\begin{array}{c}\text { Variables } \\
\text { Cuadrante }\end{array}$} & \multicolumn{3}{|c|}{ Zona 1 } & \multicolumn{3}{c|}{ Zona 2 } & \multicolumn{3}{c}{ Zona 3 } \\
\cline { 2 - 10 } & $\mathbf{T}^{\mathbf{0}} \mathbf{s}$ & $\mathbf{T}^{\mathbf{0}} \mathbf{a}$ & $\mathbf{H r}$ & $\mathbf{T}^{\mathbf{0}} \mathbf{s}$ & $\mathbf{T}^{\mathbf{0}} \mathbf{a}$ & $\mathbf{H r}$ & $\mathbf{T}^{\mathbf{0}} \mathbf{s}$ & $\mathbf{T}^{\mathbf{0}} \mathbf{a}$ & $\mathbf{H r}$ \\
\hline 1 & 26,6 & 22 & 43 & 18,9 & 22 & 50 & 19,4 & 18,1 & 65 \\
\hline 2 & 22,8 & 21 & 35 & 22,6 & 22 & 51 & 19,6 & 19 & 65 \\
\hline 3 & 24,1 & 22 & 37 & 18,7 & 21 & 51 & 20,5 & 18,8 & 65 \\
\hline 4 & 21,3 & 20 & 34 & 22,2 & 22 & 53 & 19,8 & 18,3 & 65 \\
\hline 5 & 29,4 & 21 & 36 & 23,1 & 22 & 52 & 18,7 & 18,2 & 65 \\
\hline
\end{tabular}

Se observa una diferencia marcada entre la humedad relativa registrada en la zona 1 (Tinjacá) y la zona 3 (Tota), lo cual es en gran medida debido a la influencia que ejerce la laguna de Tota en la segunda zona, originando un mayor nivel de precipitaciones, mientras que la zona de Tinjacá que está influenciada por el clima seco de la zona de Villa de Leyva, las precipitaciones son menores y así mismo su humedad relativa.

Tabla 4. Coeficiente de Correlación de Spearman con probabilidad $>[R]$, bajo una Ho: Rho $=0$ con $\mathrm{N}=25 . \mathrm{T}^{\circ} \mathrm{s}$ : temperatura del suelo;

$\mathrm{T}^{\circ} \mathrm{a}$ : temperatura ambiente; Hr: Humedad relativa

\begin{tabular}{|c|c|c|c|c|c|c|}
\hline \multirow{4}{*}{ 芯 } & \multicolumn{5}{|c|}{ OBSERVADOS } & \multirow[b]{2}{*}{ Cuadrante } \\
\hline & Observado & $\mathrm{T}^{0} \mathrm{~s}$ & $\mathrm{Hr}$ & Especies & $\mathrm{T}^{\mathrm{o}} \mathrm{a}$ & \\
\hline & 1,00000 & 0,16573 & 0,15626 & $-0,14205$ & 0,13976 & $-0,00474$ \\
\hline & 0,0 & 0,4285 & 0,4557 & 0,4982 & 0,5052 & 0,9821 \\
\hline \multirow{2}{*}{ 芯 $N$} & 1,00000 & $-0,42428$ & $-0,24188$ & $-0,23833$ & $-0,20341$ & $-0,15068$ \\
\hline & 0,0 & 0,0345 & 0,2441 & 0,2513 & 0,3294 & 0,4722 \\
\hline \multirow{2}{*}{ స్ } & 1,00000 & $-0,37199$ & $-0,16432$ & $-0,15519$ & $-0,04793$ & - \\
\hline & 0,0 & 0,0671 & 0,4325 & 0,4589 & 0,8200 & - \\
\hline
\end{tabular}


La mayor abundancia de isópodos terrestres en la localidad de Tibasosa, se debe posiblemente a una amplia variedad de microhábitats, y a una alta disponibilidad de recursos alimentarios (Rushton y Hassall, 1983), como hortalizas y leguminosas. Del mismo modo la composición y ensamblaje de plantas dicotiledóneas son fundamentales en la abundancia de isópodos (Rushton y Hassall, 1983), por que mantienen mejor la humedad del suelo (Souty-Grosset et al., 2005), conservando un hábitat favorable. De esta forma la presencia abundante de estos crustáceos en cultivos agrícolas puede provocar daños en la emergencia de plántulas y daños en cotiledones y tallos (Saluso, 2001). Aunque su impacto como plagas potenciales en agroecosistemas es limitado, está relacionado con especies sinantrópicas, es decir a hábitats asociadas a ambientes urbanos o intervenidos por el hombre (Paoletti y Hassall, 1999) y a cultivos en siembra directa y labranza convencional (Saluso, 2001).

La abundancia de P. scaber es debida a las condiciones propicias que ofrece Tibasosa (mayor número de microhábitats y alta disponibilidad de recursos), y a la mayor capacidad de agregación en relación a otros isópodos (Hassall et al., 2005); sin embargo cabe destacar que esta especie no es absolutamente cosmopolita y esta limitada más que otras especies a requisitos ecológicos (Hassall, 1996). A pesar de ser $P$. scaber una especie sinantrópica (Hornung et al., 2007), también se encontró en zonas no agrícolas.

A. vulgare fue la especie que se presentó en las tres localidades, probablemente debido a que es considerada una especie cosmopolita de amplia tolerancia a la desecación, más que $P$. scaber (Hassall et al., 1992), teniendo una amplia flexibilidad en la táctica reproductiva (alta variabilidad en los patrones reproductivos).
Los isópodos son macro descomponedores fundamentales en el ciclaje de nutrientes, sin embargo, se encontraron en altas densidades en cultivos de hortalizas en el municipio de Tibasosa; esto hace inferir que una o más especies pueden ser plaga potencial de importancia económica en la producción agrícola. Según García y Campos (2001), las especies sinantrópicas: Armadillidium vulgare y Porcellionides pruinosus pueden causar daños en la agricultura.

\section{CONCLUSIONES}

El estudio permitió identificar 5 especies de isópodos terrestres: Porcellio scaber, Porcellio dilatatus, Porcellionides pruinosus, Armadillidium vulgare y Armadillidium nasatum, siendo nuevos registros de ocurrencia para Boyacá.

En la localidad muestreada en Tibasosa, se encontró mayor isópodofauna, puesto que es una zona con mayor número de microhábitats y alta disponibilidad de recursos, lo cual la hace susceptible para que estos isópodos a futuro sean una plaga de importancia económica en la producción agrícola.

El microhábitat favorito de los isopodos fue plateo en plantas hortícolas, frutícolas y forestales, seguido de madera en descomposición.

La investigación indicó que en las condiciones del estudio las variables de temperatura ambiente, temperatura de suelo, humedad relativa y altitud de cada lugar no se relacionaron con el número de individuos, pero pueden influenciar el establecimiento de estas especies toda vez que son exóticas y algunas sinantrópicas. 


\section{AGRADECIMIENTOS}

Al Grupo Manejo Biológico de Cultivos - GMBC - de la Universidad Pedagógica y Tecnológica de Colombia, Tunja, por proveer las instalaciones y recursos para la realización del presente trabajo.

\section{BIBLIOGRAFÍA}

ARAUJO, P., BUCKUP, L. y BOND-BUCKUP, G.1996. Isópodos terrestres (Crustácea, Oniscidea) de Santa Catarina e Rio Grande do Sul. Serie Zoologia. 81(75):111 - 138.

GARCÍA, F. y CAMPOS. J. 2001. Biologia e controle de artrópodes de importância fitossanitária (Diplopoda, Symphyla, Isopoda), pouco conhecidos no Brasil. Revisto Biológico. 63(1/2):7-13.

GONZÁLEZ, G. 2004. Bichos bolita. Peligrosidad y condiciones predisponentes. En: http:// www.rizobacter.com/home/es/. 3 p; consulta: julio, 2013.

GREGORY, S. 2009. Woodlice and Waterlice (Isopoda: Oniscidea \& Asellota) in Britain and Ireland. First edition. Field Studies Council/ Centre for Ecology \& Hydrology. UK. 176 p.

HASSALL, M., DAVIS, R. y PROCTER, D. 1992. Lateral movements of isopods in a dune grassland, Proceedings of the European Congress of Entomology, First edition. Edit Jenser G. Amsterdam. 192 p.

HASSALL, M. 1996. Spatial variation in favourability of a grass heath as a habitat for woodlice (Isopoda: Oniscidea). Pedobiologia. 40:514 - 528.

HASSALL, M. TUCK, M. y JAMES, R. 2005. Effects of density and spatial heterogeneity on foraging behaviour and fitness correlates of $A r-$ madillidium vulgare (Isopoda: Oniscidea). Ethology Ecology and Evolution. 17:233 - 247.

HOPKIN, S. P. 1991. A Key to the woodlice of Britain and Ireland. Field Studies. 7:599 - 650.

HORNUNG, E. TÓTHMÉRÉSZ, B. MAGURA, T. y VILISICS, F. 2007. Changes of isopod assemblages along an urban-suburban-rural gradient in Hungary. European Journal Soil Biology. 43:158 - 165 .

HOUSTON, W.W.K. y BEESLEY, P. L. 2002. Zoological catalogue of Australia. Ed. 19.2 ${ }^{\mathrm{a}}$. CSIRO publishing. Sydney. 433 p.

HUNTER, M. ADL, S. PRINGLE, C. y COLEMAN, D. 2003. Relative effects of macroinvertebrates and habitat on the chemistry of litter during decomposition. Pedobiologia, 47:2 - 6 .

LEISTIKOW, A., WOLFGANG, W. J. 1999. Checklist of the terrestrial isopods of the new world (Crustacea, Isopoda, Oniscidea). Revista Brasileira de Zoologia. 16(1):1 - 72.

LEISTIKOW, A. 2001. Phylogeny and biogeography of South American Crinocheta traditionally placed in the family "Philosciidae" (Crustácea: Isópoda: Oniscidea). Organism Diversity \& Evolution. 4:1 - 85.

LEISTIKOW, A. y ARAUJO, P.B. 2006. The systematic position of Benthanoscia longicaudata Lemos de Castro, 1958 (Isopoda: Oniscidea: Crinocheta). Systematics and Biodiversity. 4(3): 243 - 254.

LOPES, E.R.C. SOUZA, M. BOND-BUCKUP, G. y ARAUJO, P. B. 2005. Oniscidea diversity across three environments in a altitudinal gradients in 
northeastern Rio grande do sul, Brazil. European Journal of Soil Biology. 41:99 - 107.

MYERS, P., R. ESPINOSA, C. S. PARR, T. JONES, G. S. HAMMOND, y T. A. DEWEY. 2015. The Animal Diversity Web (online). En: http:// animaldiversity.org/accounts/Armadillidium_ nasatum/classification/, $1 \mathrm{p}$.

PAOLETTI, M. y HASSALL, M. 1999. Woodlice (Isopoda: Oniscidea): their potential for assessing sustainability and use as bioindicators. Agriculture, Ecosystems and Environment. 74:157 - 165.

QUADROS, A.F. y ARAUJO. P.B. 2007. Ecological traits of two neotropical oniscideans (Crustacea: Isopoda). Acta Zoologica Sinica. 53(2):241 - 249.

RODRÍGUEZ, A. G., ORTEGA, V. V. y TREVIÑO, F. 2014. Macrocrustáceos del Parque Nacional Cumbres de Monterrey, México: distribución y estado de conservación. Revista Mexicana de Biodiversidad. 85(1):276 - 293.

RUSHTON, P. y HASSALL, M. 1983. Food and feeding rates of the terrestrial isopod Armadillidium vulgare (Latreille), Oecologia, 57: 257 - 261.

SALUSO, A. 2001. Isópodos terrestres asociados al cultivo de soja en siembra directa. INTA EEA Paraná. Argentina. En: www.inta.gov.ar/parana/info/documentos/produccion_vegetal/ soja/evaluacion_manejo/Soja_Isopodos.pdf; consulta: junio, 2013.

SOUTY-GROSSET, C., BADENHAUSSER, I., REYNOLDS, J. D. y MOREL, A. 2005. Investigations on the potential of woodlice as bioindicators of grassland habitat quality. European Journal Soil Biology. 41:109 - 116.
VANDEL, A. 1960. Isópodos terrestres (Première partie). Faune de France 64:1 - 416.

VITERI, S. MÉNDEZ, M. RESTREPO, D. y GAONA, R. 2005. Investigación participativa hacia un manejo ecológico de la cebolla de bulbo en Cucaita y Tibasosa- Boyacá. Apuntes para un manejo ecológico serie $5.35 \mathrm{p}$. 